\title{
Characterizing the Performance of the FEI Titan Environmental Transmission Electron Microscope / Scanning Transmission Electron Microscope
}

\author{
Eric A. Stach*******, Seung Min Kim**, Dmitri N. Zakharov**, Yury Zvinevich,**** Fabio H. \\ Riberio****, Lior Miller***, Robert Colby***, Norman J. Salmon*** and Bernd Kabius***** \\ *School of Materials Engineering, **Birck Nanotechnology Center, ****School of Chemical \\ Engineering, Purdue University, West Lafayette, IN 47907 \\ *** Hummingbird Scientific, LLC, Olympia, WA 98516 \\ ***** Electron Microscopy Center, Argonne National Laboratory, Argonne, IL 60439
}

We installed the first environmental-cell version of the Titan 80/300 TEM/STEM at the Birck Nanotechnology Center at Purdue University in April of 2006. We will report a comprehensive set of performance benchmarks for the system, and describe a number of experimental modifications and additional supporting equipment we have developed to increase the overall system utility. Specifically, we will characterize the electron optical performance, discuss modifications made to the vacuum system and describe the development of optimized heating holders and gas delivery systems. These results will be presented in the scientific framework of our observations of carbon nanotube nucleation, chirality selection and growth termination.[1,2]

Testing of the instrument resolution as a function of gas type, pressure and incident voltage has been performed in order to assess the degradation of image resolution due to inelastic scattering from various gases (Figure 1). These observations allow direct determination of the effective damping envelopes for imaging in gases, and thus provide an upper limit on the achievable resolution at a given pressure. We will also describe the image performance in the high-angle annular dark field STEM mode, noting the effects of differential pumping apertures on the Z-contrast. Initial electron energy loss spectroscopy (ELNES and EXELFS) results concerning scattering from gases will be also be presented, and consideration of this approach for in-situ determination of chemical reactions will be discussed.

Working with FEI, we have installed a series of additional turbomolecular pumps to the base system, leading to an expansion in the range of available working pressures in the environmental mode. This led to an improvement in the base pressure, allowing a higher reproducibility in our experiments. We have also integrated a fully functional gas reaction system, composed of 6 mass-flow controllers, integrated with pressure controllers, residual gas analysis and computer control. The resulting system exactly mimics a standard setup for rate studies in the field of catalysis. We will demonstrate the benefits attained from linking controlled, real time observations of catalyst morphological evolution with equivalent quantitative measurements of reaction rates and x-ray spectroscopy measurements of electronic structure changes during reaction.

Finally, we will show how this optimized environmental TEM has played a key role in recent discoveries concerning nanotube nucleation, chirality selection and growth termination. We will emphasize how these real time observations are most effective at providing key insights if made in a broader framework, and in close collaboration with scientists pursuing more standard approaches. 
References

[1] P.B. Amama, et al., Nano Letters, 9(1), 44-49. 2009; S.M. Kim, et al., J. Phys. Chem. Lett., in press, 2010.

[2] A.R. Harutyunyan, et al., Science, 326, 116, 2009.
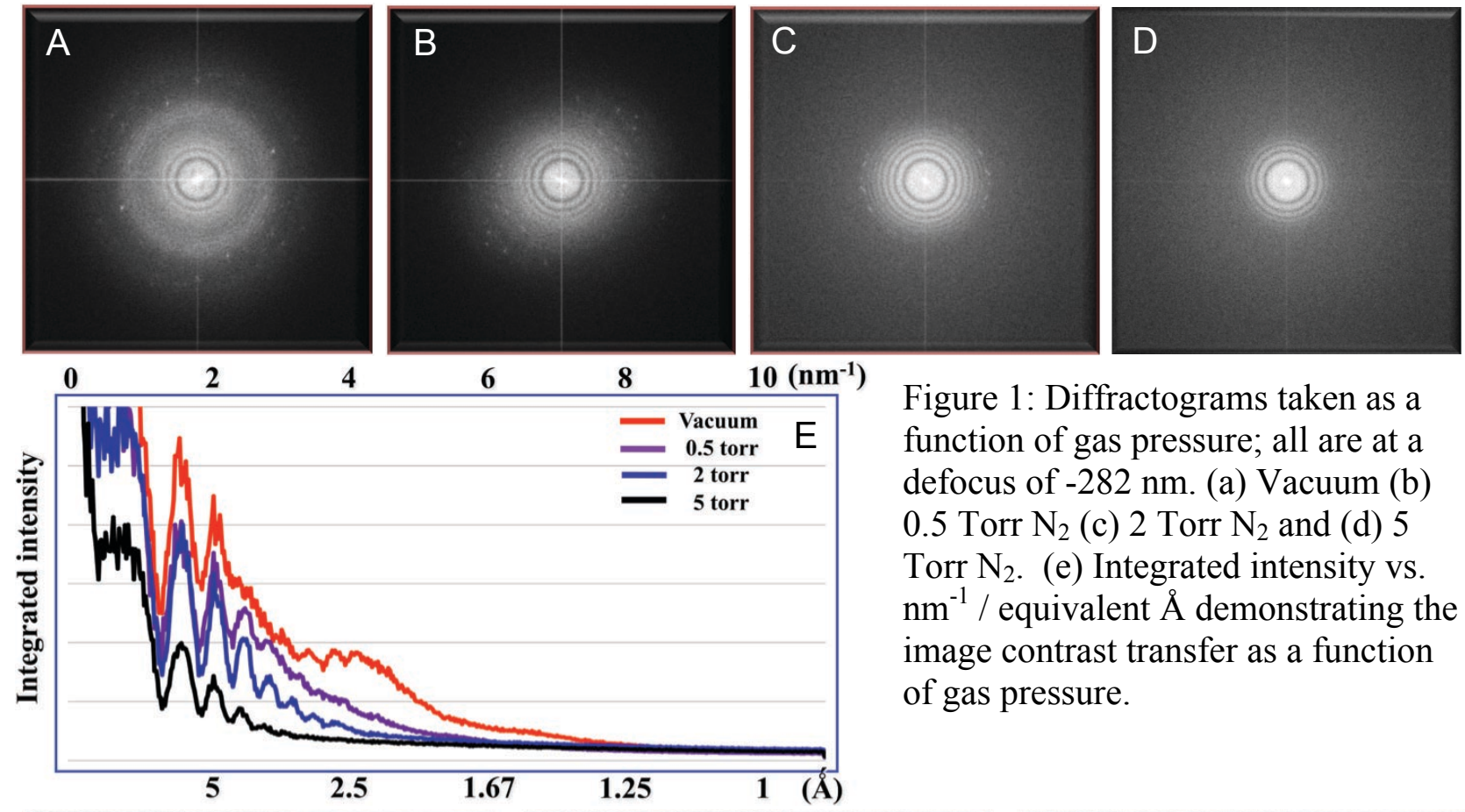

Figure 1: Diffractograms taken as a function of gas pressure; all are at a defocus of $-282 \mathrm{~nm}$. (a) Vacuum (b) 0.5 Torr $\mathrm{N}_{2}$ (c) 2 Torr $\mathrm{N}_{2}$ and (d) 5 Torr $\mathrm{N}_{2}$. (e) Integrated intensity vs. $\mathrm{nm}^{-1} /$ equivalent $\AA$ demonstrating the image contrast transfer as a function of gas pressure.
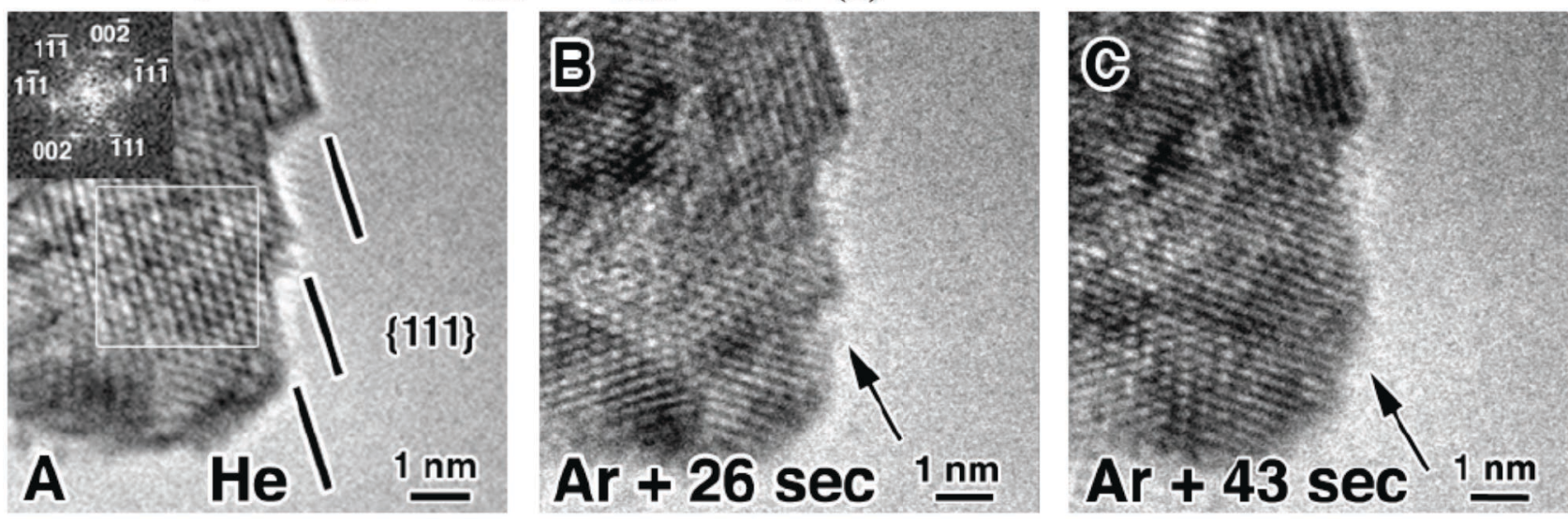

Figure 2: High-resolutiong images of the effect of gas ambient on the Fe nanoparticles used to nucleation carbon nanotubes. The images demonstrate a roughening transistion upon change (a) from a background gas of He to (b-c) a background gas of Ar. Adapted from Ref 2. 